\title{
Early Detection of Melanoma using Color and Shape Geometry Feature
}

\author{
${ }^{1,2}$ T.Y Satheesha, ${ }^{3} \mathrm{D}$ Satyanarayana and ${ }^{2}$ M.N Giri Prasad \\ ${ }^{1}$ Electronics and communication engineering, Nagarjuna College of Engineering and Technology, \\ Bangalore - Karnataka, India. \\ ${ }^{2}$ Electronics and communication engineering, Jawaharlal Nehru Technological University Anantapur, \\ Anantapuramu -Andrapradesh, India. \\ ${ }^{3}$ Electronics and communication engineering, Ragiv Gandi Memorial College of Engineering and \\ Technology, Nandyala-Andrapradesh, India. \\ ty.satish@gmail.com, dsn2003@rediffmail.com, mahendragiri1960@gmail.com
}

\begin{abstract}
Melanoma occurrence rates contain be increasing for the earlier 3 decades. The majority folks analyzed with non-melanoma carcinoma contain higher prospects to cure, however malignant melanoma endurance rates are low compare to different carcinoma varieties. It is important that one in 5 Americans will grow skin cancer in their life, and generally, one American expires from skin cancer each hour. A system to obviate this kind of skin cancer is being scheduled and is very in-demand. Initial detection of melanoma is one of the key factors to increment the chance of remedy significantly. Malignant melanomas are asymmetrical and have aberrant borders with rages and notched edges, thus analyzing the form of the skin lesion is consequential for melanoma early detection and aversion. In this paper, we have a tendency to introduce an automatic skin lesion segmentation and analysis for premature detection and obviation predicated on color and shape geometry. The system additionally incorporates extra feature sets such as color to find the wound type. In our planned system, we use PH2 Dermoscopy image information. This image info contains a complete of fifty dermoscopy pictures of lesions, together with traditional, malignant melanoma and atypical cases. Our approach of analyzing the form pure mathematics and therefore the color are going to be subsidiary to detect atypical lesions afore it grows and becomes a melanoma case.
\end{abstract}

Keywords: skin cancer, image segmentation, melanoma, classification.

\section{Introduction}

\subsection{Background and Motivation}

Nowadays carcinoma has been progressively known because the major explanation for deaths. carcinoma may be classified into 3 major types: skin cancer, basal cell cancer (BCC), and epithelial cell carcinomas (SCC). Among these 3 sorts, skin cancer begins from melanocytes and crack the danger scale because it produces rapidly and metastasizes rapidly. BCC initiates from basal cells and it's the smallest amount dangerous variety of carcinoma. SCC begins from squamous cells and produces with a slower rate than

DOI: 10.14738/jbemi.24.1315

Publication Date: $14^{\text {th }}$ July 2015

URL: http://dx.doi.org/10.14738/jbemi. 24.1315 
melanomas however matures metastasis at some later stages. Although the majority diagnosed with carcinoma contain higher probabilities to treat, melanoma endurance rates are lesser than that of nonmelanoma skin cancer. As additional latest cases of carcinoma area unit being analyze within the U.S. every year, an automatic system to help within the interference and early detection is extremely indemand. Following area unit the estimations of the yank Cancer Society for malignant melanoma within the us for the year 2014 [1]: around seventy six, 100 new melanomas area unit to be diagnosed (about forty three, 890 in men and thirty two, 210 in women). Around nine, 710 fatalities area unit expected as a results of malignant melanoma (about vi, 470 men and three, 240 women). For thirty years, additional or less, malignant melanoma rates are increasing steady. It's twenty times additional common for White race to own malignant melanoma than in African-Americans. In general, throughout the life, the chance of obtaining malignant melanoma is around two ( 1 in 50$)$ for whites, $0.1 \%(1$ in 1,000) for blacks, and $0.5 \%$ (1 in 200) for Hispanics.

\subsection{Contribution}

In this paper, we have a tendency to propose the parts of a completely unique smart-phone-based time period system to help within the carcinoma hindrance and early detection. The projected malignant melanoma lesion segmentation algorithmic program is conferred during this paper. A system to stop this kind of carcinoma is being expected and is very in-demand, as a lot of new cases of malignant melanoma area unit being analyzed within the U.S. each year. In our projected system, the user is capable to capture the photographs of skin moles in addition as sure environmental metrics (e.g. ultraviolet radiation). One part of our system is a picture process module to classify beneath that class the moles fall into; traditional, atypical, or malignant melanoma. AN alert are provided to the user to hunt medical facilitate if the mole belongs to the atypical or malignant melanoma class.

The outcome of this technique is mean to assist users to prevent developing carcinoma by triggering a period alert that notices the users to restrict exposure to harmful actinic ray radiation. The system also will enable users to capture pictures of skin lesions and analyze it at time period. Users are ready to have associate early detection of skin cancer that increases the probabilities of self-made treatment.

\section{Related Work}

Skin image identification on good phones has become one among the engaging and hard to please analysis areas within the past few years. Karargyris et al. have worked on a complicated image-processing mobile application for observation carcinoma [2]. The authors bestowed associate application for skin interference employing a mobile device. a cheap accent was used for up the standard of the pictures. in addition, a complicated computer code framework for image process backs the system to investigate the input pictures. Their pictures info was little, and consisted of solely half-dozen pictures of traditional cases and half-dozen pictures of suspicious case.

Doukas et al. developed a system consisting of a mobile application that might gain and acknowledge moles in skin pictures \& categorise them consistent with their brutality into skin cancer, blemish and kind lesions. As indicated by the conducted check, Support Vector Machine (SVM) resulted in mere seventy seven.06\% classification accuracy [3]. 
T.Y Satheesha, D Satyanarayana and M.N Giri Prasad; Early Detection of Melanoma using Color and Shape Geometry Feature. Journal of Biomedical Engineering and Medical Imaging, Volume 2, No 4, August (2015) , pp 33-45

Massone et al. introduced mobile tele dermoscopy; melanoma diagnosing by one click. The system provide a service designed towards management of patients with growing skin disorder or for follow-up with patients requiring systematic treatment. Tele dermoscopy enabled transmission of dermoscopic pictures through e-mail or explicit web application. This system lacked an automatic image processing module and was altogether dependable on the availability of medical specialist to diagnose and classify the dermoscopic pictures. Hence, it's not thought of a time period system[4].

Wadhawan et al. projected a transportable documents for skin cancer recognition on hand-held devices supported the accepted bag-of-features framework [5]. They showed that the foremost machine intensive and time overwhelming algorithms of the library, specifically image segmentation and image classification, can do accuracy and speed of execution similar to a personal computer. These findings incontestable that it's potential to run subtle medical specialty imaging applications on sensible phones and different handheld devices, that have the advantage of movability and low price, and thus, will build a big impact on health health care delivery as helpful devices in underserved and remote areas. However, their system didn't enable the user to capture pictures mistreatment the sensible phone.

Ramlakhan et al. [6] introduced a mobile automatic skin lesion organization. Their system consisted of 3major components: image segmentation, feature calculation, and classification. Experimental result showed that the system wasn't extremely economical, achieving a median accuracy of sixty six.7\%, with average malignant category recall/sensitivity of sixty. $7 \%$ and specificity of eighty.5\%. Barata et al. projected 2systems for the recognition of malignant melanoma cases in dermoscopy pictures victimization color and texture options [7]. This paper aimed toward decisive the simplest system for skin lesion classification. The authors compared the role of color and texture options in lesion classification and determined that set of options were a lot of discriminative. it absolutely was all over that color options vanquish texture options once used alone which each strategies bring home the bacon excellent results, i.e., sensitivity =ninety six and Specificity = eightieth for international strategies (i.e. international options color and texture) against Sensitivity $=100 \%$ and Specificity $=$ seventy fifth for native strategies (i.e. native options color and texture). However, this technique didn't run on a sensible phone and didn't enable the users to capture skin pictures. 


\section{Proposed System}

The flow of proposed system as shown in figure 1.
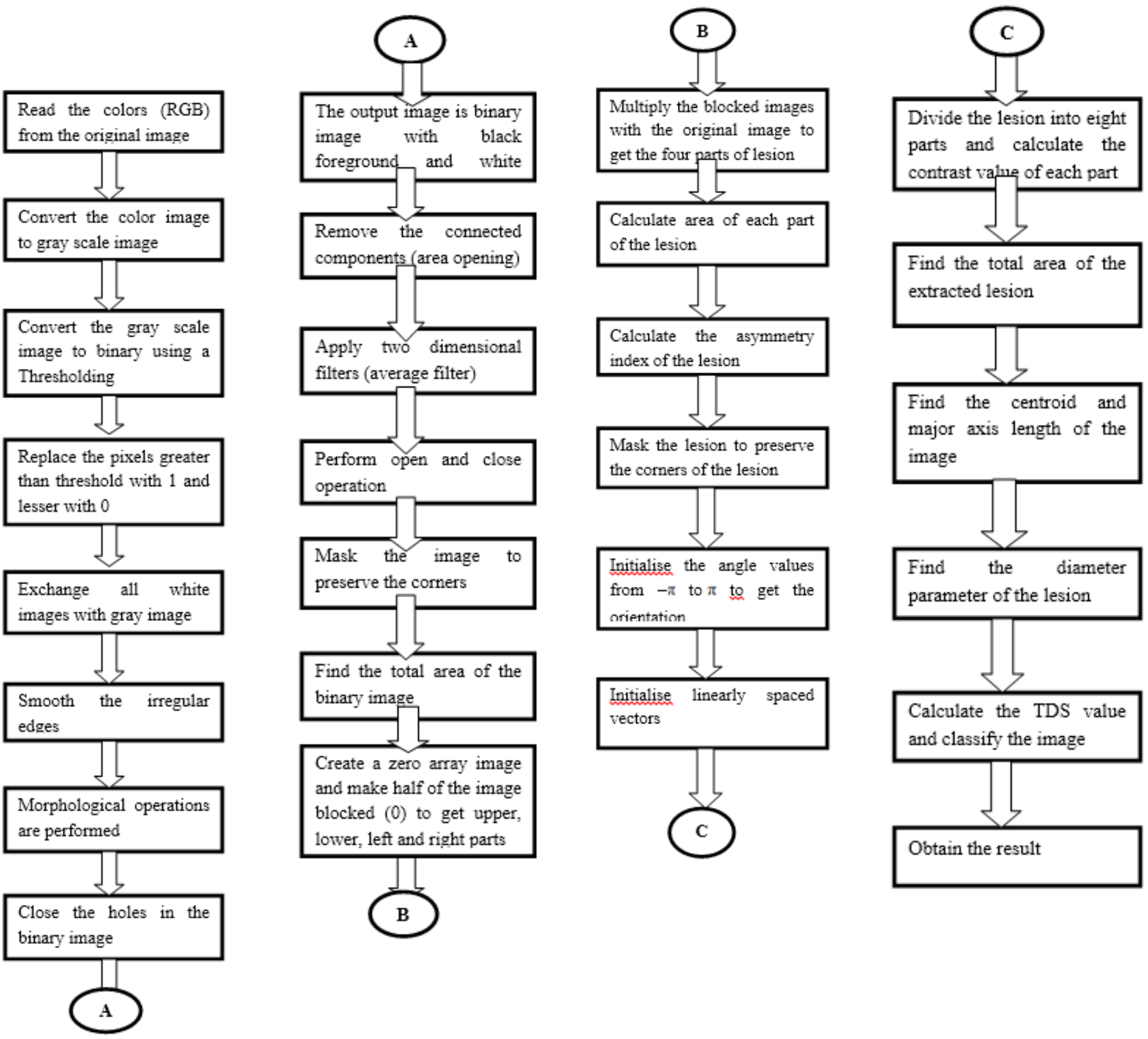

Figure 1: Flow chart

\subsection{Pre-Processing}

The original color image is a combination of RGB color so from the original image we extract the RGB channel images which are shown in figures 2 respectively. 


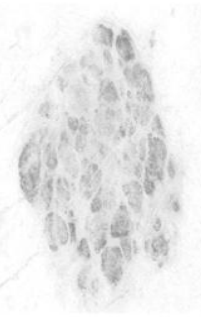

Figure-a: Red channel image

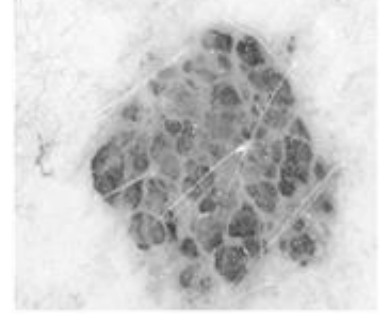

Figure-b: Red channel image

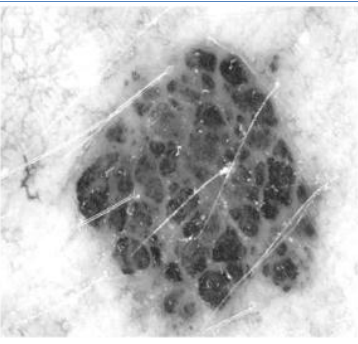

Figure-c: Red channel image

Figure 2: primary colors images

After extracting the RGB channel images we extract the blue channel image for processing since it is having more information. Then to enhance the quality of the image the histogram equalization is performed. The figure $3(a)$ shows the histogram equalized image in which the brightness values are assigned across the pixels of image here the intensity is distributed.

Take the original color image and then convert the original color image into gray scale image this can be done by using rgb2gray command. By using this command the color images are converted into gray scale images. Here the blue channel histogram equalized image is converted into gray scale image as shown in figure $3(b)$.

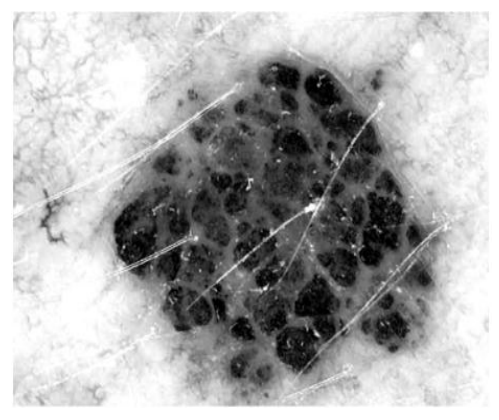

Fig a: Histogram equalized image

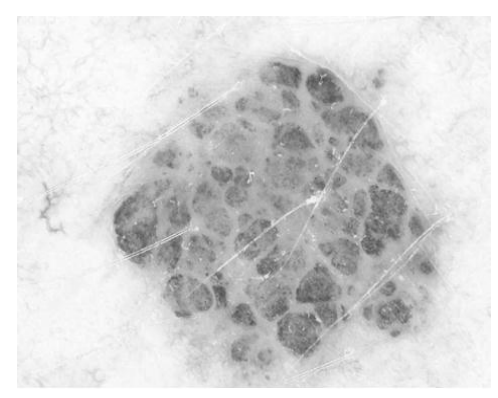

Fig b: Gray scale image

Figure 3: final preprocessed image

\subsection{Segmentation}

Melanoma lesion segmentation to divide the lesion from the background is a vital step before beginning with the options extraction so as to classify the 3 differing kinds of lesion (i.e. normal, melanoma and atypical). The segmentation steps are followed by: initially, we have a tendency to browse the RGB (i.e. color image) dermoscopy image and convert to grey scale image. we have a tendency to convert Red, Green and Blue values to grey scale values by developing a weighted add of the $R, G$, and $B$ parts (i.e. $0.2989 * \mathrm{R}+0.5870 * \mathrm{G}+0.1140 * \mathrm{~B})$.

Then, we tend to produce a 2 dimensional filter of the Average low pass filter kind. We tend to used Equations one and a couple of to make the Average filter [10]. In Equation a pair of, $\mathrm{h}$ may be a 2 dimensional filter of size $9 \times 9$. Next, we tend to filter the image with the third-dimensional filter $h$. Step a pair of in Figure a pair of shows the impact of mistreatment the Average low pass filter.

After the Average filter is applied, we have a tendency to calculate a worldwide threshold which will be accustomed convert associate degree intensity image to a binary image. We have a tendency to used Otsu's methodology [11] that chooses the brink to reduce the intra class variation of the white and black 
pixels. This methodology was derived from the read purpose of the discriminating analysis. This directly deals with the matter of evaluating the goodness of thresholds. Associate degree best threshold is chosen by the discerning criterion.

The next step is to translate the grey scale image to binary image exploitation the calculated threshold. To do this, we tend to replaced all pixels within the grey scale image with brightness level larger than threshold with the worth zero (black), and replaced all alternative pixels with the worth one (white).

The next step is to get rid of the white corners within the dermoscopy image. So as to try to to this, we have a tendency to mask the resulted image within the previous step with mask1. All white pixels within the corners are replaced with black pixels.

After applying the brink, the sides of the output image become irregular. To sleek the sides, we have a tendency to initial produce a disk-shaped structure component. The technique we have a tendency to accustomed produce the disk structure component is termed radial decomposition victimization periodic lines $[12,13]$. The disk structure component is made to protect the rounded nature of the lesion. We have a tendency to nominal the radius as eleven pixels in order that the biggest gap gets crammed. Then, we have a tendency to use the disk structure component to perform a morphological shut operation on the image. The morphological shut operation could be a dilation followed by erosion; we have a tendency to use identical disk structure component for each operations.

Next, we tend to apply the morphological open operation on the binary image. The morphological open operation is attrition followed by dilation; we tend to used identical disk structure component that we tend to created within the previous steps for each operations

In the next step, we tend to used Associate in nursing rule supported morphological reconstruction [14] so as to fill the holes within a binary image. The hole may be a gaggle of background pixels that cannot be reached by filling inside the background from the sting of the image.

In the next step, we tend to applied AN algorithmic rule supported active contour [15] to section the grayscale image. The active contour algorithmic rule fragments the 2-D grayscale image into foreground (lesion) and background areas victimization effectively outline the primarily based segmentation. The active contour perform uses the image shown in Figure two, step seven as a mask in specifying the initial state of the active contour. This algorithmic rule uses the Sparse Field level-set methodology [16] for developing active contour evolution. In addition stops the event of the active contour if the contour position among the present iteration is that an equivalent united of the contour positions from the foremost recent 5 iterations, or if the most variety of iterations (i.e. 400) has been accomplished. The output image could be a binary image wherever the foreground is white (logical true) and also the background is black (logical false).

Small objects are removed in the next step. To achieve this, first found the connected components. Secondly, area of each component was computed. Lastly, all small objects having fewer than 50 pixels are removed. This operation is called area opening.

Finally we have a tendency to use the disk structure part that we have a tendency to create within the previous steps to perform a morphological shut and open operation. 
T.Y Satheesha, D Satyanarayana and M.N Giri Prasad; Early Detection of Melanoma using Color and Shape Geometry Feature. Journal of Biomedical Engineering and Medical Imaging, Volume 2, No 4, August (2015) , pp 33-45

After converting the image to gray scale image the segmentation of the image can be done, The Otsu's method of thresholding is applied by using command "Graythresh". The Otsu method calculates the global threshold value which is used to translate a gray scale image into binary image. The threshold lies in the range between 0, 1.After calculating threshold "im2 bw" is used to convert all the pixel values above threshold to 1 (white) and the values below threshold to 0 (black). Now the image is converted to binary image. Resultant image is shown in figure 4(a). After converting the image to binary, the holes that are present in the region of skin lesion are filled. The command "imfill" performs this operation. This process is called morphological reconstruction. The holes filled image is shown in figure 4(b). After filling the holes the small objects i.e. connected components in the image needs to be removed. "Bwareaopen" performs this operation. Figure 4(c) shows area opened image. Once the connected components are removed then the image needs to be filtered. Here the average filter is used to filter the image. The "fspecial" creates a various 2 Dimensional filters. The average filter removes impulse noise in the image. The average filtered image is shown in figure 4(d). After filtering the boundary of the image is traced. The "Bwboundaries" performs this operation. It traces the external boundaries of the lesion and also the holes if any in the image. This can be done to detect the outer border of the segmented image. The segmented boundary detected image is shown in figure $4(e)$.

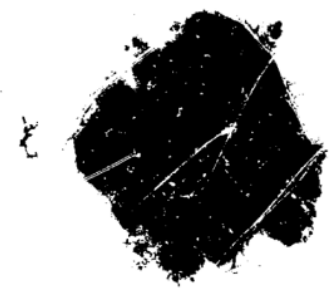

Figure (a). Binary converted image

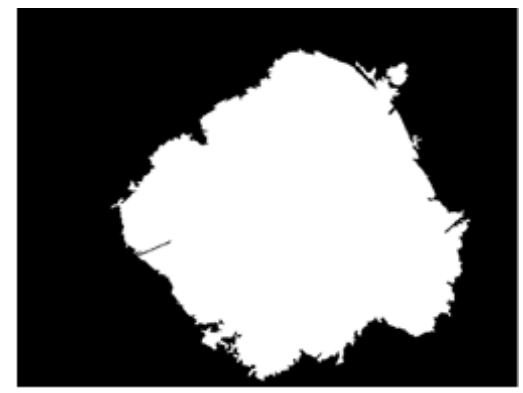

Figure (c). Connected components removed (area opening)

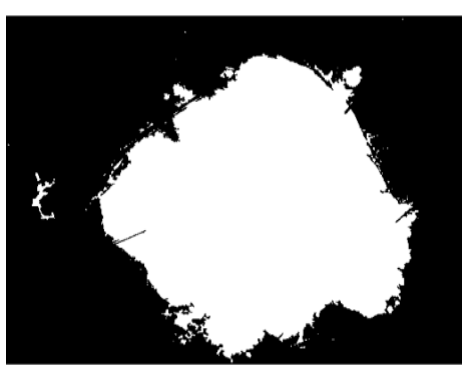

Figure (b). Holes filled image (morphological reconstruction)

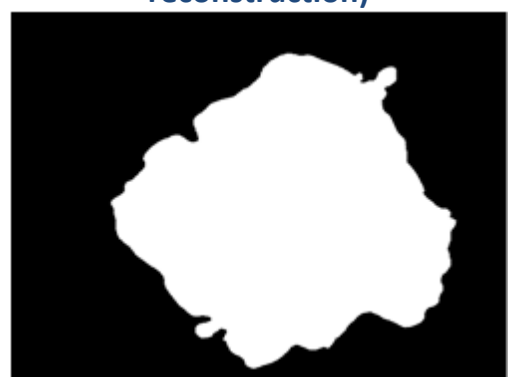

Figure (d). Filtered image (average filtering) 

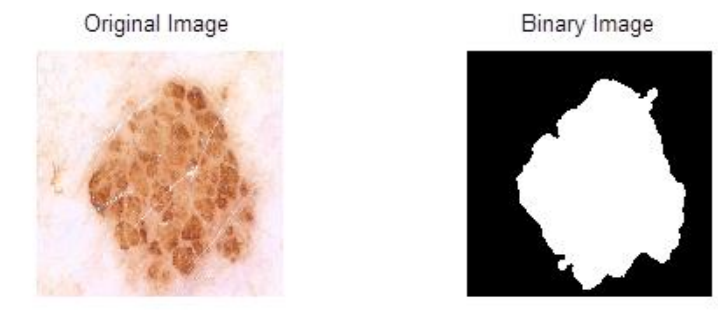

Melonoma Segmented Original Image

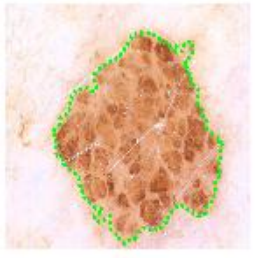

Fig e. Final Segmented images

Figure 4: Segmented Image

\subsection{Feature Extraction}

After the segmentation is done the Feature extraction is performed. The feature extraction involves the extraction of four features such as Asymmetry, Border irregularity, Color, and Diameter. A rule called $A B C D$ rule is used to perform this feature extraction.

The asymmetry index of the image is calculated by calculating the total area and the area of the lesion. To total area of the image is calculated by using "region props" to calculate the area we find the centroid and the major axis length. After area is calculated the image is rotated. The original image is divided into four parts. To divide the image we create a zero array image and then the array values are converted into logical values .then half of the created image is made 0 (blocked) and is done to four halves or four times. Then the blocked images are multiplied with original image to get the upper, lower right and left parts of the region of interest. The area of the each lesion is calculated and the asymmetry parameter is fond shown in figure $5(a)$.

Upper Part ROI

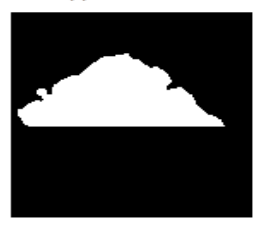

Left Part ROI

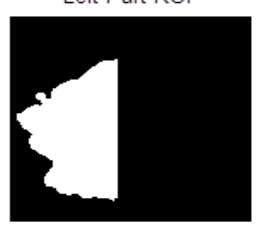

Lower Part ROI

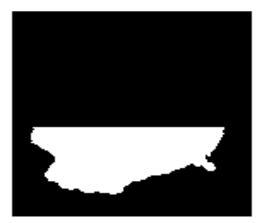

Right Part ROI

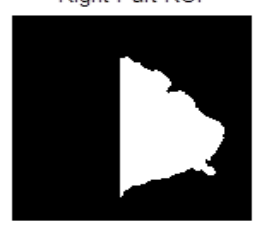

Figure (a). Asymmetry feature 
The image is masked along the boundary to preserve the corners of the lesion. The masked image is shown in figure 5(b). The figure 5(c) shows the original boundary image after masking. The borders of the inventive image is shown in figure $5(\mathrm{~d})$. The angle values from $-\pi$ to $\pi$ are initialised and the linearly spaced vectors are initialised which generates a row of equally spaced vectors between two points and the image borders are divided into eight parts as shown in figure 5(e).

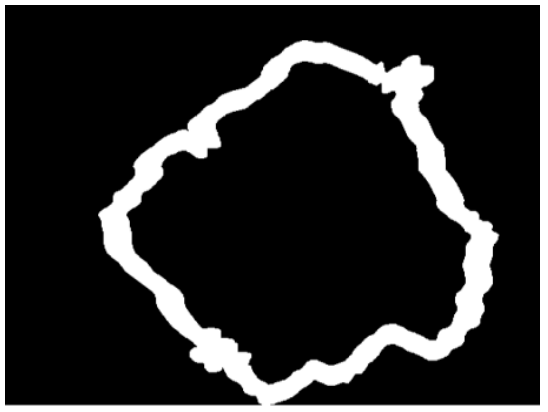

Figure (b). Original boundary masked image

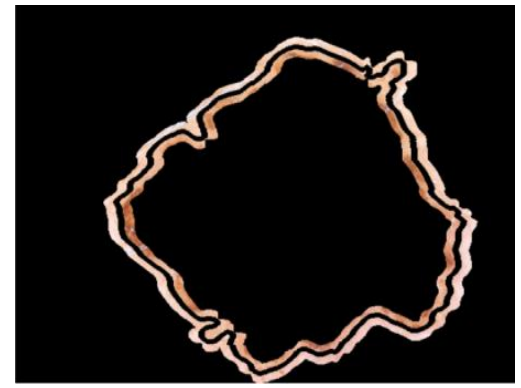

Figure (d) Border of original image

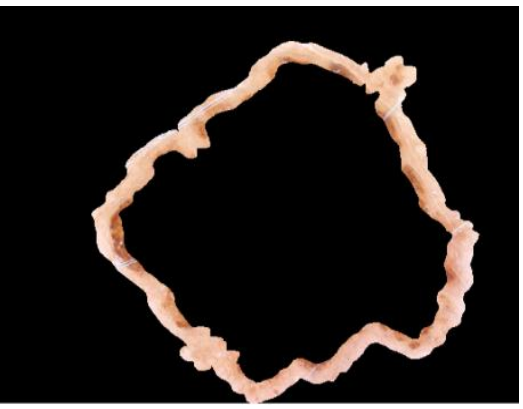

Figure (c) Original boundary image

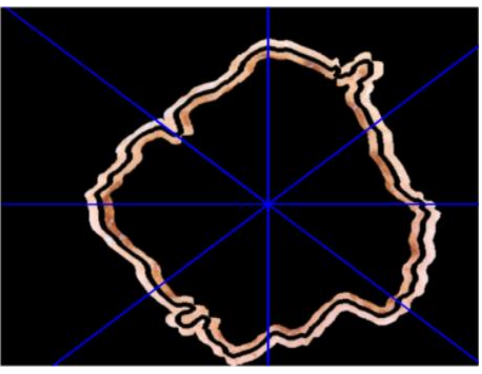

Figure $€$ Feature parameter 2

The divided border parts are shown in figure $5(f)$.
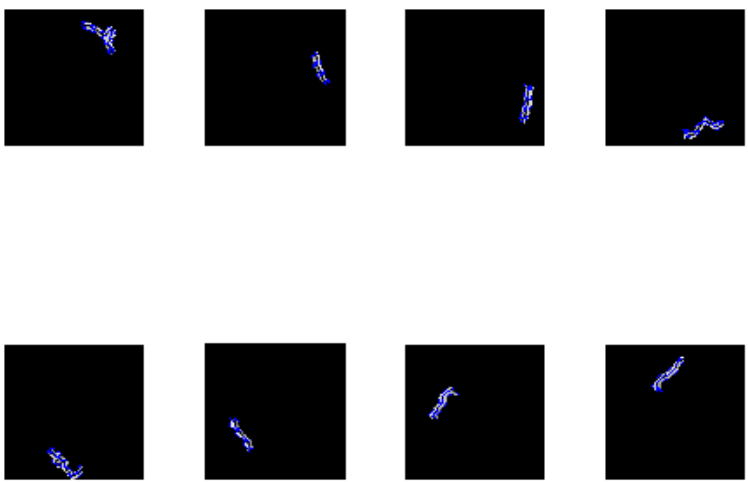

Figure (f) borders of blue channel image

Then the contrast at each part of the image is calculated and the extracted original contrast image is shown in figure $5(\mathrm{~g})$. The total area of the image is calculated by using the command bwarea. The diameter of the lesion is calculated by finding the row and column size of the lesion. Then the total ratio is found and then the centroid and the major axis length of the lesion are calculated. By using these values the 
diameter of the lesion is found and the circle is drawn from 0 to 2 . Based on this the diameter parameter is calculated. The diameter of the image is shown in figure $5(\mathrm{~h})$.

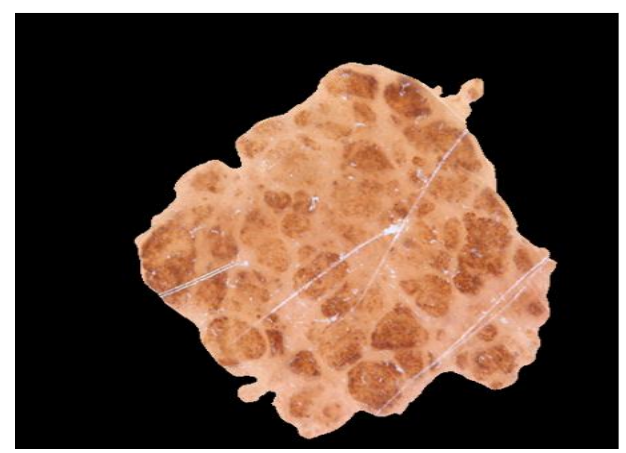

Figure (g) Extracted original image

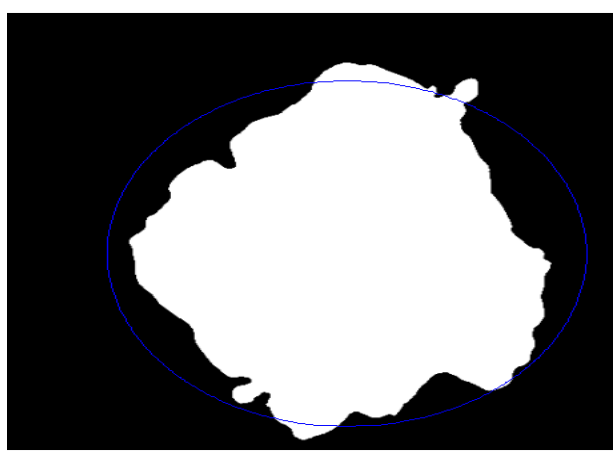

Figure (h) Diameter calculated image Figure 5: Feature extracted parts

\subsection{Classifier:}

The primary stage of this framework is to perform image process to denoise the image and to section the Region of Interest (ROI) of the skin. When we tend to section the ROI, we tend to extract the image options like 2-D quick Fourier remodel options set (FFT2), 2D distinct circular function remodel options set (DCT2), size and complexness options sets. Next, we tend to feed the extracted options to the classifiers. In this classifier just one classifier is employed to classify the ROI into 3 classes, normal, atypical or malignant melanoma. We tend to used SVM classifier.

The 2-D quick Fourier rework (FFT) feature set [18] computes the distinct Fourier rework (DFT) and its inverse. 2-D distinct circular function rework (DCT) [19] expresses a finite sequence of knowledge points in terms of a total of circular function functions periodic at totally different frequencies. As a result, 2-D FFT and 2-D DCT area unit wide used for several applications. Therefore, we tend to use such options to classify the skin cancer cases.

The 2-D FFT feature set includes the primary constituent of FFT2, the primary constituent of the crosscorrelation [20] of the primary twenty rows and columns of FFT2, the mean of the primary twenty rows and columns of FFT2, and also the variance of the primary twenty rows and columns of FFT2. The 2-D DCT feature set includes the primary constituent of DCT2, the primary constituent of the cross correlation of the primary twenty rows and columns of DCT2, the mean of the primary twenty rows and columns of DCT2 and also the variance of the primary twenty rows and columns of DCT2. The quality options set includes the image ROI's mean, variance and mode supported the intensity info. For the color options set we tend to calculated the 3D bar chart of the parts of the laboratory color model. so as to urge the 2nd color bar chart from the 3D color bar chart, we tend to accumulate all values within the illumination axis. As a result, we tend to get $8 \times 8=64$ color bins, every thought of joined features.

After finding all the four parameter values the Total Dermatoscopic Value (TDS) is calculated to classify the images into Normal, Melanoma and Malignant melanoma cases. The final evaluation result is as shown in table(1). 
T.Y Satheesha, D Satyanarayana and M.N Giri Prasad; Early Detection of Melanoma using Color and Shape Geometry Feature. Journal of Biomedical Engineering and Medical Imaging, Volume 2, No 4, August (2015) , pp 33-45

Table (1): classifier matrix

\begin{tabular}{|c|c|c|c|}
\hline Parameters & Normal & Atypical & Melanoma \\
\hline Normal & 90.1 & 9.7 & 0.2 \\
\hline Atypical & 7.2 & 92.3 & 0.5 \\
\hline Melanoma & 0.1 & 9.4 & 90.5 \\
\hline
\end{tabular}

\section{Conclusion and Future Work}

The incidence of malignant melanoma has arisen in recent years dramatically. Fast and effective detection methods are desperately needed to save thousands of lives each year. It has been known for many years that most skin cancers, including melanoma, are curable if treated early. They have been discussed many algorithms for the detection of different characteristics "ABCD" of early malignant melanoma. For $A=$ Asymmetry, asymmetry remains undefined until now. Dermatologists consider only symmetric tumour almost symmetrical and there is no absolute division between symmetrical and non-symmetrical tumour yet. There is still much disagreement among doctors in making tumours is moderately asymmetrical. The asymmetry is a very critical feature in the detection of malignant melanoma, an asymmetry index is calculated here to determine symmetry parameter, the method is simple and fast; which it is designed based on the geometric feature of a flat shape.

Color segmentation was used in this work for the shape of the tumour. With major axes are skew it is determined around the axis of symmetry nearby comparing the absolute area to the total area of the shape differences. The results in this paper have shown that this method is suitable; in fact, test results have been clinically shown fairly high success rate. For B = Border Irregularity, the border of the tumour was obtained edge detection in gray scale. Then a general measure for detecting irregularities border to be the geometric mean measurement and the results obtained were in agreement with the tumours found. It was also said that a combination of viewing goodness borders and other such measure will be helpful in making decisions. Thus, the decision of whether the border is irregular or cannot be performed more accurately. For $\mathrm{C}=$ Color Variegation, the results of this work demonstrate the success of color image segmentation using uniform color spaces. Therefore, this color segmentation algorithm is successful as an aid in the search for tumour border and different colors in a tumour; Moreover, you can be successful as an aid in finding ulcer or reflections. This interpretation color spaces of color is more closely related to human perception, as it has been responsible for the nonlinear response of luminance. Along with the color difference formulas, this space is very useful in the accurate assessment of perceptual closeness between two colors. For $\mathrm{D}=$ Diameter generally greater than $6 \mathrm{~mm}$, since the slides used in reality, the result of the classification of this work is not complete because only three early clinical features of malignant melanoma are being studied.

A classified "non-melanoma" can be found to be a melanoma tumour after it has gone through the analysis of size, so size analysis should not be omitted. After all, the best way to prevent ourselves from this disease is to take care of ourselves carefully, to keep away from the sun, and keep our bodies as healthy as possible. It is better and easier to prevent than to find a cure. According to research conducted by the Canadian Association of Dermatology, it found that the majority of skin cancers can be prevented. Moreover, the understanding of us, to be observable, and remain alert to pigmented spots on the skin.

In this paper we have a tendency to given the parts of a system to help within the melanoma interference and early detection. We have a tendency to planned an automatic segmentation algorithmic program and 
a completely unique framework to classify the dermoscopic pictures into traditional, atypical and malignant melanoma. Future work can concentrate on shaping and extracting novel options, for instance pigment network, to enhance the accuracy of classification.

\section{REFERENCES}

[1] American Cancer Society, Cancer Facts \& Figures. Available: http://www.cancer.org/research/cancerfactsstatistics/cancerfactsfigures2014/index

[2] A. Karargyris, et.al "DERMA/care: An advanced image-processing mobile application for monitoring skin cancer," in Tools with Artificial Intelligence (ICTAI), 2012 IEEE 24th International Conference on, 2012, pp.1-7.

[3] C. Doukas, et.al "Automated skin lesion assessment using mobile technologies and cloud platforms," in Engineering in Medicine and Biology Society (EMBC), 2012 Annual International Conference of the IEEE, 2012, pp. 2444-2447.

[4] C. Massone, et. al "Mobile tele dermoscopy-melanoma diagnosis by one click?," in Seminars in cutaneous medicine and surgery, 2009, pp. 203-205.

[5] T. Wadhawan, et, al "SkinScan (C: A portable library for melanoma detection on handheld devices," in Biomedical Imaging: From Nano to Macro, 2011 IEEE International Symposium on, 2011, pp. 133-136.

[6] K. Ramlakhan and Y. Shang, "A Mobile Automated Skin Lesion Classification System," in Tools with Artificial Intelligence (ICTAI), 2011 23rd IEEE International Conference on, 2011, pp.138141.

[7] C. Barata, et, al , "Two Systems for the Detection of Melanomas in Dermoscopy Images Using Texture and Color Features," Systems Journal, IEEE, vol. 99, pp. 1-15, 2013.

[8] M. Poulsen, et al., "High-risk Merkel cell carcinoma of the skin treated with synchronous carboplatin/etoposide and radiation: a Trans- Tasman Radiation Oncology Group Study-TROG 96: 07," Journal of Clinical Oncology, vol. 21, pp.4371-4376, 2003.

[9] M. Ichihashi, M. et al., "UV-induced skin damage," Toxicology, vol. 189, pp. 21-39, 2003.

[10] K. Ito and K. Xiong, "Gaussian filters for nonlinear filtering problems," Automatic Control, IEEE Transactions on, vol. 45, pp. 910-927, 2000.

[11] N. Otsu, "A threshold selection method from graylevel histograms," Automatica, vol. 11, pp. 2327, 1975. 
T.Y Satheesha, D Satyanarayana and M.N Giri Prasad; Early Detection of Melanoma using Color and Shape Geometry Feature. Journal of Biomedical Engineering and Medical Imaging, Volume 2, No 4, August (2015) , pp 33-45

[12] R. Jones and P. Soille, "Periodic lines: Definition, cascades, and application to granulometries,"Pattern Recognition Letters, vol. 17, pp. 1057- 1063, 1996.

[13] R. Adams, "Radial decomposition of disks and spheres," CVGIP: Graphical Models and Image Processing, vol. 55, pp. 325-332, 1993.

[14] P. Soille, Morphological image analysis: principles and applications: Springer-Verlag New York, Inc., 2003.

[15] T. F. Chan and L. A. Vese, "Active contours without edges," Image processing, IEEE transactions on, vol. 10, pp. 266-277, 2001.

[16] R. T. Whitaker, "A level-set approach to 3D reconstruction from range data," InternationalJournal of Computer Vision, vol. 29, pp. 203-231, 1998.

[17] T. Mendonca, P. M. Ferreira, J. S. Marques, A. R. Marcal, and J. Rozeira, "PH 2-A dermoscopic image database for research and benchmarking," in Engineering in Medicine and Biology Society (EMBC), 2013 35th Annual International Conference of the IEEE, 2013, pp. 5437-5440.

[18] J. S. Walker, Fast fourier transforms vol. 24: CRC press, 1996.

[19] G. Strang, "The discrete cosine transform," SIAM review, vol. 41, pp. 135-147, 1999.

[20] R. D. Keane and R. J. Adrian, "Theory of cross correlation analysis of PIV images," Applied scientific research, vol. 49, pp. 191-215, 1992. 